\title{
Cerebellar Disorders-At the Crossroad of Molecular Pathways and Diagnosis
}

\author{
Mario Manto • Daniele Marmolino
}

Published online: 27 October 2009

(C) Springer Science + Business Media, LLC 2009

\begin{abstract}
Our understanding of the pathogenesis of cerebellar ataxias has started several decades ago and is continuously growing. The numerous mechanisms of cerebellar dysfunction are being discovered by numerous groups of researchers worldwide. Neuronal damage results from a complex interaction of metabolic pathways, which leads to symptoms observed in cerebellar disorders. The main mechanisms at the molecular level are the following: impairment of DNA repair and replication, deregulation of transcription/deficits of processing/transport of RNA, abnormal protein transport and misfolding, aggregates both at the nuclear and cytosolic level, activation of caspases, apoptosis, involvement of autophagic mechanisms, oxidative stress and mitochondrial dysfunction, excitotoxicity, abnormal lipid metabolism, impaired axonal transport and vesicle trafficking, and defects of the neurotransmission. The convergence of the current clinical classification with molecular findings is expected. This integration is a basic substrate for the rationale development of therapies.
\end{abstract}

Keywords Cerebellum · Ataxias · Pathogenesis · Diagnosis

M. Manto $\cdot$ D. Marmolino

Laboratoire de Neurologie Expérimentale ULB-Erasme,

Brussels, Belgium

M. Manto

FNRS,

Brussels, Belgium

M. Manto $(\square)$

FNRS-Laboratoire de Neurologie Expérimentale, ULB-Erasme,

Route de Lennik 808,

1070 Brussels, Belgium

e-mail: mmanto@ulb.ac.be
Cerebellar ataxias are being growingly recognized worldwide. Cerebellar disorders include a group of heterogeneous disorders with noticeable overlap between entities, including sporadic and hereditary ataxias [1,2]. These latter are often divided into recessive ataxias, dominant ataxias, ataxias related to a mitochondrial disorder which affect oxidative phosphorylation in most cases, and X-linked ataxias. Dominant ataxias include the wide spectrum of socalled spinocerebellar ataxias (SCAs/ADCA: autosomal dominant cerebellar ataxias, divided in several types according to Harding's classification). The most common diseases are summarized in Table 1. A diagnostic tree is proposed to the reader (Fig. 1).

Major breakthroughs in our understanding of the pathogenesis of cerebellar ataxias have been reached thanks to technical advances and the elaboration of relevant cellular and animal models, these latter reproducing to various extents human brain disorders. At the molecular level, the main discoveries of these last 25 years in terms of pathogenesis of cerebellar disorders can be grouped as follows in subcategories:

1. Deficits in DNA repair

2. Transcriptional deregulation

3. Accumulation of toxic proteins or decreased clearance of proteins

4. Misfolding

5. Caspase activation

6. Apoptosis

7. Primary or secondary mitochondrial dysfunction

8. Oxidative stress

9. $N$-methyl-D-aspartate (NMDA)-mediated excitotoxicity and $\alpha$-amino-3-hydroxy-5-methyl-4-isoxazolepropionic acid (AMPA)-mediated excitotoxicity

10. Disturbances at the lysosomal level 
Table 1 Most common cerebellar disorders

1. Sporadic ataxias

Structural disorders - malformations

Neurodevelopmental disorders

Immune disorders (multiple sclerosis, cerebellar ataxia with anti-glutamic acid decarboxylase antibodies, gluten ataxia, Miller-Fisher syndrome, systemic lupus erythematosus, Sjögren syndrome)

Infections

Vascular disorders (stroke: ischemia, hemorrhage)

Intoxication (ethanol, drugs) and environmental causes

Ataxias of endocrine origin (thyroid dysfunction)

Primary neoplasias (medulloblastomas, astrocytomas, ependymomas, meningiomas, lymphomas, histiocytosis) and metastases

Paraneoplastic ataxia (anti-Yo, anti-Hu, anti-Ri, anti-Tr, anti-CV2 antibodies)

Idiopathic late-onset ataxia

Cerebellar form of multiple system atrophy (cMSA)

Superficial siderosis

Migraine

2. Genetic ataxias

Recessive disorders

Friedreich ataxia (FA)

Vitamin E deficiency

Ataxia with vitamin E deficiency (AVED)

Abetalipoproteinemia (ABL)

Cholestatic liver disease

Intestinal resection

Ataxia with ocular motor apraxia (AOA1, AOA2)

Refsum disease (RD)

Cerebrotendinous xanthomatosis (CTX)

Early onset cerebellar ataxia with retained reflexes (EOCARR)

Ataxia-telangiectasia (AT) and related disorders

Deficiency in Coenzyme Q10

Autosomal-recessive spastic ataxia of Charlevoix-Saguenay (ARSACS)

Marinesco-Sjögren syndrome (MSS)

Wilson's disease

Aceruloplasminemia

Niemann-Pick type C

Late-onset Tay-Sachs disease (LOTS)

ARCA

Ataxia with hypogonadism

Ataxia and optic atrophy (Behr syndrome)

Progressive myoclonic epilepsies

Karak syndrome

Spinocerebellar ataxia with axonal neuropathy (SCAN1)

Infantile-onset spinocerebellar ataxia (IOSCA)

Mitochondrial recessive ataxia syndrome (MIRAS;

POL $\gamma$ mutations)

Xeroderma pigmentosum

Hartnup disease
Table 1 (continued)

\author{
Maple syrup disease \\ Carnitine acetyltransferase deficiency \\ Hydroxyglutaric aciduria \\ Congenital forms \\ Joubert syndrome \\ Cayman ataxia \\ $\mathrm{COACH}$ syndrome \\ Pontocerebellar hypoplasia \\ Ataxic cerebral palsy \\ Dominant disorders \\ Spinocerebellar ataxias (SCAs, 1-30) \\ Pure cerebellar syndrome (SCA5, 6, 11, 26) \\ Cerebellar ataxia-plus \\ Episodic ataxias (EAs, EA1-7) \\ Dominantly inherited spastic ataxia syndromes \\ Spastic paraplegia, ataxia, mental retardation (SPAR) \\ Ataxia/spasticity with congenital miosis \\ Myelocerebellar syndrome \\ Cerebellar ataxia, deafness and narcolepsia \\ Essential tremor (sporadic forms exist also) \\ Von Hippel-Lindau syndrome (VHL) \\ Mitochondrial disorders \\ Kearns-Sayre Syndrome \\ May-White Syndrome \\ MNGIE (ophthalmoparesia, peripheral neuropathy, and \\ gastrointestinal symptoms), Leigh syndrome \\ NARP (neuropathy, ataxia, and retinitis pigmentosa) \\ MELAS (mitochondrial encephalomyopathy, lactic acidosis \\ with stroke-like episodes) \\ MEERF (myoclonus epilepsy with ragged red fibers). \\ $X$-linked ataxias \\ Fragile-X tremor ataxia syndrome \\ Arts syndrome \\ X-linked adrenoleukodystrophy (X-ALD) \\ Rett syndrome \\ Ataxia-dementia \\ Ataxia-Deafness syndrome \\ Sideroblastic anemia and spinocerebellar ataxia $(\mathrm{ABC} 7)$ \\ Deficit in pyruvate dehydrogenase E1-alpha \\ Pelizaeus-Merzbacher allelic variant
}

11. Abnormal fatty acid metabolism

12. Disorders of neurotransmission

Figure 2 illustrates these pathways. A main field of investigations is on SCAs. Currently, 28 SCAs are identified [3]. In several SCAs, the expansion of $\mathrm{CAG}$ tracts in certain genes results in transcription of proteins with abnormally long polyQ (polyglutamine) inserts [4]. Such a mechanism is shared with Huntington's disease 
Fig. 1. Diagnostic algorithm of cerebellar disorders. The assessment of a patient with a suspicion of a cerebellar disorder is illustrated. Abbreviations: EA episodic ataxias, $S C A$ spinocerebellar ataxia (previously identified as $A D C A$ autosomal dominant spinocerebellar ataxia), $F A$ Friedreich ataxia, LOFA late-onset Friedreich ataxia, FARR Friedreich ataxia with retained tendon reflexes, $V L C F A$ very long-chain fatty acid, $E E G$ electroencephalography, EP/NCV evoked potentials, nerve conduction velocities, $C S F$ cerebrospinal fluid, FXTAS fragile $\mathrm{X}$ tremor/ataxia syndrome, MSA/sOPCA multiple system atrophy/sporadic olivopontocerebellar atrophy, ILOCA idiopathic late-onset cerebellar atrophy, CJD: CreutzfeldtJakob disease, GSS GerstmannSträussler-Scheinker disease

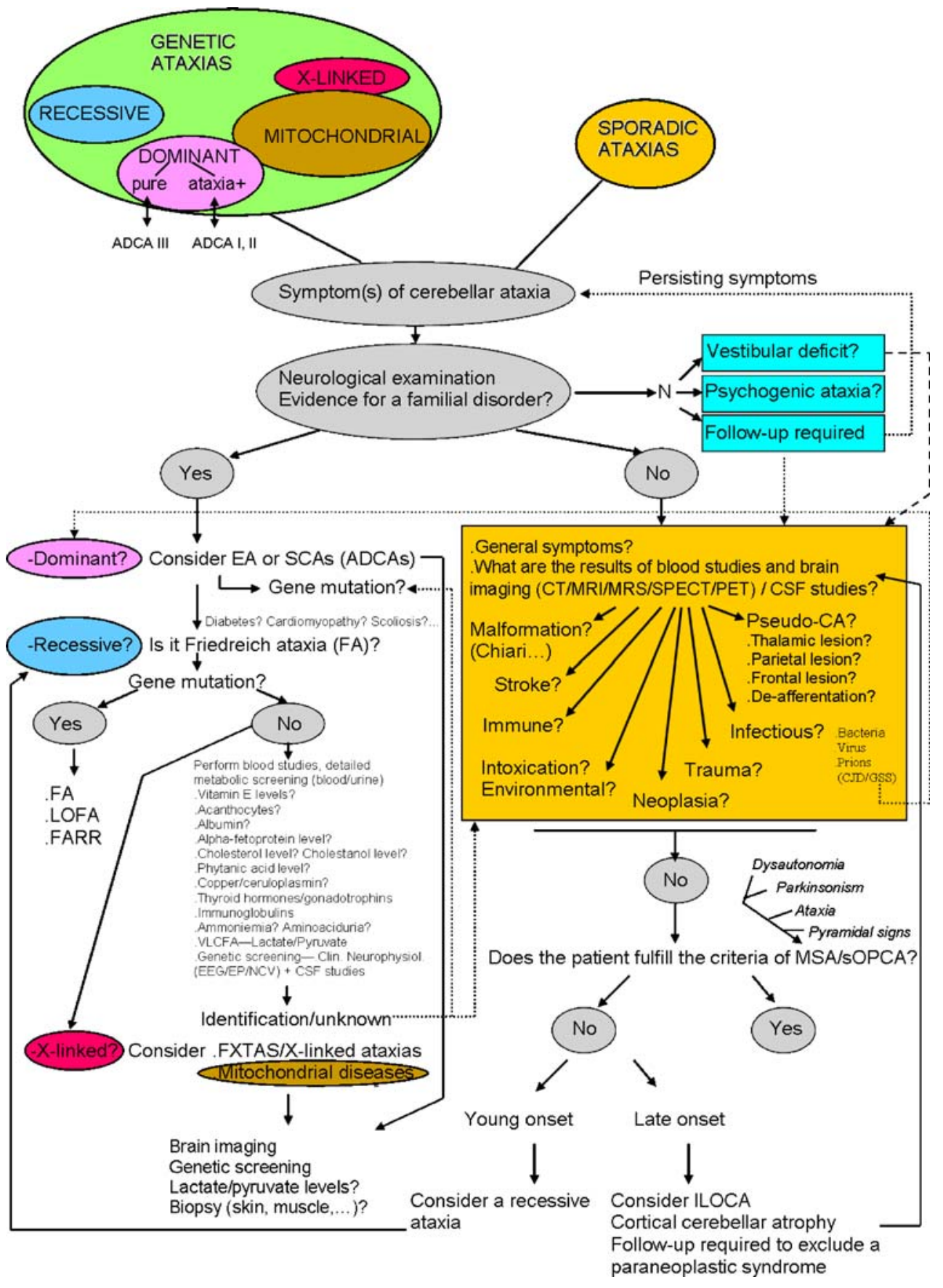

(HD) and spinal bulbar muscular atrophy (SBMA). Proteins form aggregates which contribute to neuronal loss. Expanded polyQ tracts participate in the formation of partially unfolded proteins [4]. Neurological deficits of polyQ repeat disorders appear for expanding tracts exceeding the critical value of 35-45 glutamines [4, 5]. Several models have been developed to recapitulate the molecular mechanisms occurring in cerebellar disorders, ranging from in vitro studies to complex animal models, each of them having advantages and disadvantages for translational research [6]. In vitro experiments indicate that proteasomes are unable to digest
polyQ tracts [7]. Aggregates sequester proteasomes, ubiquitin, polyQ fragments and recruit HSP70 [7]. Therefore, essential proteins are depleted from the cells, an event which contributes to cell vulnerability [7]. Expanded polyglutamine tracts decrease the dimerization of TBP (TATA box protein) and enhance the binding to the transcription factor TFIIB [3]. The consecutive depletion of TFIIB is another factor causing neural susceptibility. SCA7 is an example of cerebellar disorder associated with defects of acetylation/deacetylation [8]. Mutant ataxin-7 targets the process of chromatin remodeling, causing 


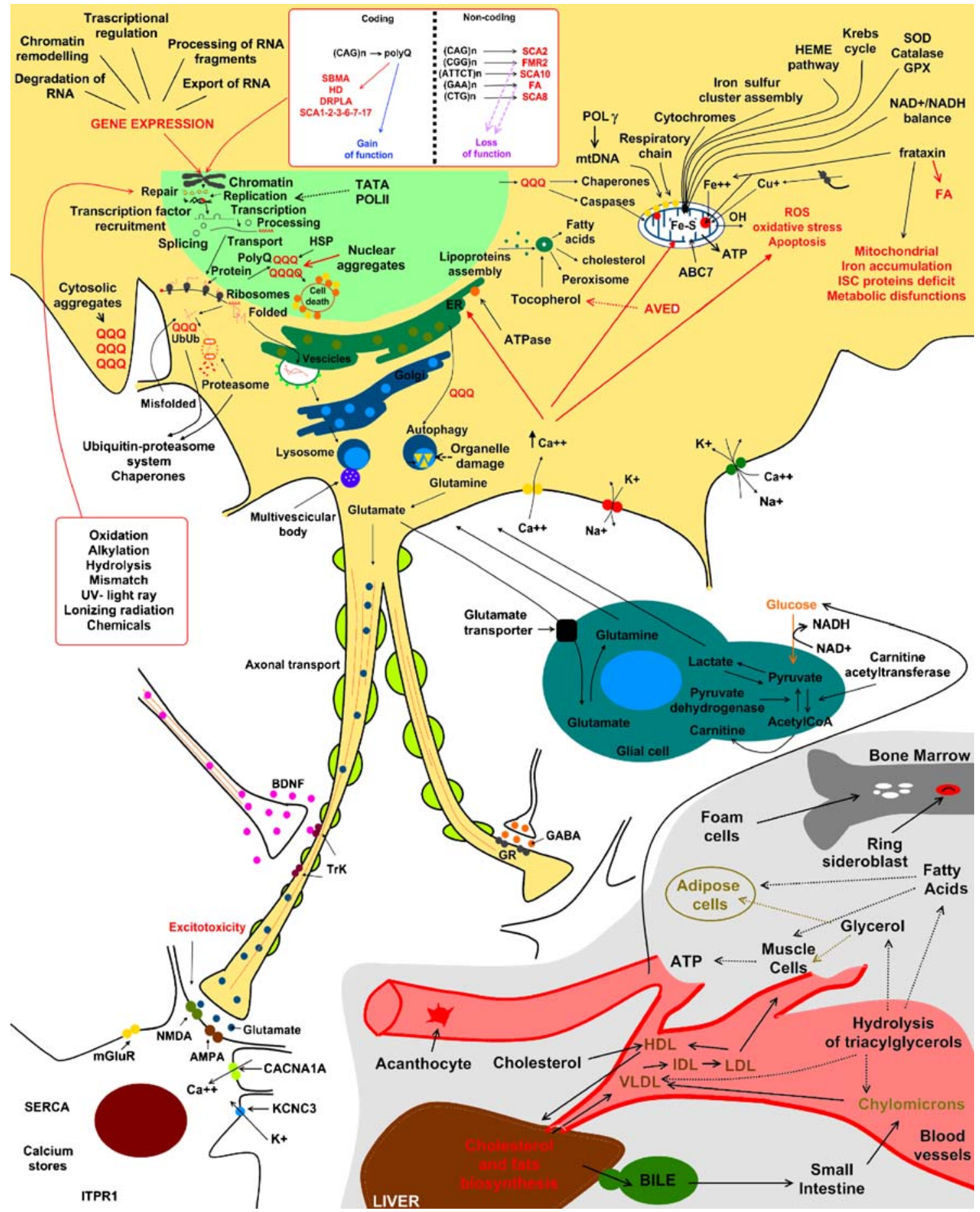

transcriptional down-regulation. Ataxin-7's normal function as a transcription factor may explain the selective vulnerability of specific neuronal populations in SCA7 [9]. Other proteins, such as ataxin-1, involve both transcriptional regulation and RNA splicing [3]. Ataxin-2 interferes with the metabolism of RNA as a result of an interaction at the level of polyribosomes [10]. Ataxin-3 is also implicated in transcriptional deregulation. The protein acts as a repressor of transcription [3]. Ataxin-3 is closely linked to the phenomenon of ubiquitination/deubiquitination [11]. Protein cleavage is caspase-dependent and this cleavage boosts neuronal loss, suggesting that therapies targeting the cleavage of ataxin-3 might slow the disease progression in SCA3 [12].

Impairment of neurotransmission is another mechanism shared by several cerebellar disorders. Impairment of glutamatergic/calcium signaling has been pointed in various sporadic and genetic ataxias, including SCA6, SCA13, or 
4 Fig. 2. Molecular pathways involved in hereditary cerebellar ataxias and which contribute to DNA/RNA/protein defects ending in cell death. Several neurons are illustrated, as well as a glial cell. The various pathophysiological processes may involve some specific categories of cells, according to the disease considered. In some disorders, the molecular pathogenesis still remains to be elucidated. In some instances, organelles are represented in association with a specific disorder, when this latter is critically linked to the given intracellular element, such as mitochondria for Friedreich ataxia $(F A)$. Keymechanisms affect DNA, RNA, apoptosis, impairment of proteasome degradation, calcium homeostasis, mitochondrial dysfunction, axonal transport, trafficking of vesicles, excitotoxic cascades/oxidative damage, dysfunction of ion channels, deficits of neurotransmission. Abbreviations: SBMA spinal bulbar muscular atrophy, HD Huntington's disease, DRPLA dentatorubral-pallidoluysian atrophy, FMR2 fragile $\mathrm{X}$ mental retardation gene, TATA TATA box binding protein, $P O L I I$ RNA polymerase II, $H S P$ heat shock proteins, $U b$ ubiquitin; Mitochondria: Poly polymerase-gamma, mtDNA mitochnondrial DNA, SOD superoxide dismutase, GPX glutathione peroxidase, $A B C 7$ ATP-binding cassette 7, AVED ataxia with vitamin E deficiency, $C A C N A 1 A$ voltage-dependent calcium channel $\mathrm{P} / \mathrm{Q}$ type (alpha $1 \mathrm{~A}$ subunit); KCNC3 potassium voltage-gated channel (subfamily $\mathrm{C}$ member 3), SERCA Sarco/endoplasmic reticulum $\mathrm{Ca}^{2+}$-ATPase, ITPRI inositol 1,4,5-triphosphate receptor (type 1), HDL highdensity lipoprotein, $V L D L$ very low-density lipoprotein, $I D L$ intermediate-density lipoprotein

SCA27. SCA6 is associated with a CAG expansion in the CACNA1A subunit of the voltage-gated calcium channel $\mathrm{P} / \mathrm{Q} \mathrm{CaV} 2.1$. Increase in calcium signaling contributes to a gain-of-function, as well as translocation of a fragment to the nucleus [3, 13]. The glutamate transporter EAAT4 is stabilized by beta-III spectrin, whose mutations cause SCA5 [14]. Decreased reuptake of glutamate contributes to the cascade of NMDA and AMPA-mediated excitotoxicity [15]. Further studies are needed to confirm that SCA20 is also related to an overflow of glutamate $[3,16]$. Mutations of the KCNC3 voltage-gated potassium channel (SCA13) affect directly the depolarization of neuronal membranes.

We are at the beginning phase of the integration of the clinico-genetic classification with molecular pathways involved in the pathogenesis of each family or subtype of cerebellar disorders. A reshaping of the clinico-genetic classification taking into account the molecular discoveries is likely to emerge soon. This fusion would have important consequences in terms of management, therapies, and future investigations in cerebellar research. In most cases, therapies of devastating ataxic disorders have remained purely symptomatic, because of (1) the lack of understanding of the molecular deficits, (2) the inability to identify the critical pathway(s) for each disorder, and (3) as a result of the use of models of cerebellar disorders which have brought non-relevant information for the development of therapies. Several therapeutic lines have now been drawn: modulation of gene expression and transcriptional tuning, changing the clearance of a mutant protein, making the protein less toxic, action upon chaperones, use of transglutaminase inhibitors, effects on caspase activation, effects on mitochondrial function and oxidative damage [17]. Development of therapies of sporadic ataxias might take an unexpected benefit from these investigations. The reverse is also true. For instance, there is hope that looking beyond the traditional mechanism of glutamate-receptordriven excitotoxicity could be very useful in stroke models [18]. There are growing evidence that ionic imbalance contributes to cell death; hence, the ongoing studies on sodium-calcium exchangers, hemichannels, volumeregulated anion channels, acid-sensing channels, transient receptor potential channels, nonselective cation channels, and signaling cascades [18]. New insights in the molecular pathogenesis of sporadic and hereditary ataxias open also now doors towards the unraveling of the myriad of secrets of the cerebellar circuits.

Acknowledgements M.M. is supported by the FNRS-Belgium, D.M. is supported by the Fonds Erasme-Belgium.

\section{References}

1. Brusse E, Maat-Kievit JA, van Swieten JC (2007) Diagnosis and management of early- and late-onset cerebellar ataxia. Clin Genet 71(1):12-24

2. Klockgether $\mathrm{T}$ (2008) The clinical diagnosis of autosomal dominant spinocerebellar ataxias. Cerebellum 7(2):101-105

3. Carlson KM, Andresen JM, Orr HT (2009) Emerging pathogenic pathways in the spinocerebellar ataxias. Curr Opin Genet Dev 19 (3):247-253

4. Barton S, Jacak R, Khare SD, Ding F, Dokholyan NV (2007) The length dependence of the polyQ-mediated protein aggregation. J Biol Chem 282(35):25487-25492

5. Ross CA, Wood JD, Schilling G, Peters MF, Nucifora FC Jr, Cooper JK, Sharp AH, Margolis RL, Borchelt DR (1999) Polyglutamine pathogenesis. Philos Trans R Soc Lond B Biol Sci 354(1386): 1005-1011

6. Paulson HL, Pulst SM (2007) Polyglutamine ataxias: in vitro and in vivo models. In: Brice A, Pulst SM (eds) Spinocerebellar degenerations. The ataxias and spastic paraplegias. Butterworth, pp 145-169

7. Raspe M, Gillis J, Krol H, Krom S, Bosch K, van Veen H, Reits E (2009) Mimicking proteasomal release of polyglutamine peptides initiates aggregation and toxicity. J Cell Sci 122(Pt 18):32623271

8. Helmlinger D, Hardy S, Abou-Sleymane G, Eberlin A, Bowman AB, Gansmüller A, Picaud S, Zoghbi HY, Trottier Y, Tora L, Devys D (2006) Glutamine-expanded ataxin-7 alters TFTC/ STAGA recruitment and chromatin structure leading to photoreceptor dysfunction. PLoS Biol 4(3):e67

9. Garden GA, La Spada AR (2008) Molecular pathogenesis and cellular pathology of spinocerebellar ataxia type 7 neurodegeneration. Cerebellum 7(2):138-149

10. Satterfield TF, Pallanck LJ (2006) Ataxin-2 and its Drosophila homolog, ATX2, physically assemble with polyribosomes. Hum Mol Genet 15(16):2523-2532

11. Winborn BJ, Travis SM, Todi SV, Scaglione KM, Xu P, Williams AJ, Cohen RE, Peng J, Paulson HL (2008) The deubiquitinating 
enzyme ataxin-3, a polyglutamine disease protein, edits Lys63 linkages in mixed linkage ubiquitin chains. J Biol Chem 283 (39):26436-26443

12. Jung J, Xu K, Lessing D, Bonini NM (2009) Preventing Ataxin-3 protein cleavage mitigates degeneration in a Drosophila model of SCA-3. Hum Mol Genet. Sep 25. In press

13. Kordasiewicz HB, Thompson RM, Clark HB, Gomez CM (2006) C-termini of $\mathrm{P} / \mathrm{Q}$-type $\mathrm{Ca} 2+$ channel alpha1A subunits translocate to nuclei and promote polyglutamine-mediated toxicity. Hum Mol Genet 15(10):1587-1599

14. Ikeda Y, Dick KA, Weatherspoon MR, Gincel D, Armbrust KR, Dalton JC, Stevanin G, Dürr A, Zühlke C, Bürk K, Clark HB, Brice A, Rothstein JD, Schut LJ, Day JW, Ranum LP (2006) Spectrin mutations cause spinocerebellar ataxia type 5. Nat Genet 38(2):184-190
15. Slemmer JE, De Zeeuw CI, Weber JT (2005) Don't get too excited: mechanisms of glutamate-mediated Purkinje cell death. Prog Brain Res 148:367-390

16. Knight MA, Hernandez D, Diede SJ, Dauwerse HG, Rafferty I, van de Leemput J, Forrest SM, Gardner RJ, Storey E, van Ommen GJ, Tapscott SJ, Fischbeck KH, Singleton AB (2008) A duplication at chromosome 11q12.2-11q12.3 is associated with spinocerebellar ataxia type 20. Hum Mol Genet 17(24):3847-3853

17. Underwood BR, Rubinsztein DC (2008) Spinocerebellar ataxias caused by polyglutamine expansions: a review of therapeutic strategies. Cerebellum 7(2):215-221

18. Besancon E, Guo S, Lok J, Tymianski M, Lo EH (2008) Beyond NMDA and AMPA glutamate receptors: emerging mechanisms for ionic imbalance and cell death in stroke. Trends Pharmacol Sci 29(5):268-275 